\title{
Expression of Somatostatin Receptor 2 in Somatotropinoma Correlated with the Short-Term Efficacy of Somatostatin Analogues
}

\author{
Wenjuan Liu, ${ }^{1,2}$ Lina Xie, ${ }^{3}$ Min He, ${ }^{1,2}$ Ming Shen, ${ }^{4,5}$ Jingjing Zhu, ${ }^{6}$ Yeping Yang, ${ }^{1,2}$ \\ Meng Wang, ${ }^{1,2}$ Ji Hu, ${ }^{7}$ Hongying Ye, ${ }^{1,5}$ Yiming Li, ${ }^{1,2,5}$ Yao Zhao, ${ }^{4,5}$ and Zhaoyun Zhang ${ }^{1,2,5}$ \\ ${ }^{1}$ Division of Endocrinology and Metabolism, Huashan Hospital, Fudan University, Shanghai 200040, China \\ ${ }^{2}$ Institute of Endocrinology and Diabetology, Fudan University, Shanghai 200040, China \\ ${ }^{3}$ Department of Endocrinology, Kunshan Rehabilitation Hospital, Suzhou, Jiangsu 215314, China \\ ${ }^{4}$ Department of Neurosurgery, Huashan Hospital, Fudan University, Shanghai 200040, China \\ ${ }^{5}$ Shanghai Pituitary Tumor Center, Shanghai 200040, China \\ ${ }^{6}$ Department of Pathology, Huashan Hospital, Fudan University, Shanghai 200040, China \\ ${ }^{7}$ Department of Endocrinology, The Second Affiliated Hospital, Soochow University, Suzhou, Jiangsu 215004, China
}

Correspondence should be addressed to Zhaoyun Zhang; zhaoyunzhang@fudan.edu.cn

Received 28 November 2016; Accepted 23 January 2017; Published 15 March 2017

Academic Editor: Andrea G. Lania

Copyright (C) 2017 Wenjuan Liu et al. This is an open access article distributed under the Creative Commons Attribution License, which permits unrestricted use, distribution, and reproduction in any medium, provided the original work is properly cited.

\begin{abstract}
The expression of somatostatin receptor subtypes (SSTRs) in pituitary growth hormone- (GH-) secreting adenomas may predict the response to somatostatin analogues (SSA). Our aim was to evaluate the value of the immunohistochemical (IHC) scores of 2 subtypes, SSTR2 and SSTR5, in predicting the short-term efficacy of SSA therapy in patients with active acromegaly. Ninetythree newly diagnosed acromegalic patients were included in our study. These patients were categorized into either a SSApretreated group (SA, $n=63$ ) or a direct-surgery group (DS, $n=30$ ), depending on whether or not presurgical SSA treatment was received. IHC analysis, using a 12-grade scoring system, with rabbit monoclonal antibodies against SSTR2 and SSTR5, was performed on all adenoma tissues. The reduction of GH, IGF-1, and tumor size after treatment with SSA for 3 months was measured. Compared with that in the DS group, SSTR2 expression was lower in the SA group. Additionally, in the SA group, SSTR2 expression was positively correlated with the reduction of IGF-1 and tumor volume. However, there was no correlation between the SSTR5 score and the efficacy of SSA. In conclusion, the protein expression of SSTR2, but not of SSTR5, is a valuable indicator in predicting biochemical and tumor size response to short-term SSA treatment in acromegalic patients.
\end{abstract}

\section{Introduction}

Somatostatin analogues (SSA) are the most widely used medications for the treatment of active acromegaly [1]. They exert biological effects by binding to $G$ proteincoupled receptors known as somatostatin receptors (SSTR), mainly SSTR2 and SSTR5. The overall efficacy of SSA is $\sim 35 \%$ in biochemical remission rate (mean GH levels $<2.5 \mu \mathrm{g} / \mathrm{L}$ and IGF-1 normalization) $[2,3]$ and $\sim 70 \%$ in oncological response rate (tumor volume shrinkage $>20 \%$ ) $[4,5]$. Therefore, it is advantageous to identify patients who may respond to SSA.
Clinical and experimental studies have thus far been focused on predictors related to SSA response such as age, gender, GH levels, T2-weighted signal intensity on MRI, indium-111 octreotide scanning, and SSTR subtype expression patterns $[2,3,5-10]$. However, different studies have shown conflicting results which cannot be translated directly to clinical practice $[5,11,12]$.

The predictive value of SSTR expression is still a matter of controversy. Previous studies have been focused on SSTR2 and SSTR5, as octreotide binds to these two receptors with high affinity [13]. However, only SSTR2 was reported to be associated with the acute and long-term effects of octreotide 
TABLE 1: Basic characteristics of the cohorts.

\begin{tabular}{|c|c|c|c|c|}
\hline & Entire cohort & DS group & SA group & $P$ value \\
\hline Number (female/male) & $93(59 / 34)$ & $30(20 / 10)$ & $63(39 / 24)$ & $0.82^{\mathrm{a}}$ \\
\hline Age at diagnosis, y & $41 \pm 12$ & $41 \pm 13$ & $43 \pm 12$ & $0.59^{\mathrm{b}}$ \\
\hline $\mathrm{GH}, \mu \mathrm{g} / \mathrm{L}$ & $34.2(13.5-57.9)$ & $26.4(12.1-66.5)$ & $40.9(15.7-56.1)$ & $0.22^{\mathrm{c}}$ \\
\hline IGF-1 index & $2.9(2.4-3.6)$ & $2.4(1.9-2.8)$ & $3.0(2.6-3.6)$ & $<0.01^{\mathrm{c}}$ \\
\hline Adenoma volume $(n=77), \mathrm{mm}^{3}$ & $1900(1000-2515)$ & $1470(249-6160)(n=19)$ & $2110(1000-2500)(n=58)$ & $0.65^{\mathrm{c}}$ \\
\hline
\end{tabular}

DS, direct surgery group; SA, pretreatment with somatostatin analogues before surgery; GH, growth hormone; IGF-1, insulin-like growth factor 1. Data are mean \pm SEM for age at diagnosis and median with interquartile range for GH, IGF-1 index, and adenoma volume. For adenoma volume, there are 77 patients available for analysis (for DS group, $n=19$, while for SA group, $n=58$ ). ${ }^{\text {a }} X^{2}$ test. 'Student's $t$-test. 'Student's $t$-test after log transformation.

in most studies $[9,14,15]$. In addition, data about the predictive value of SSTR in the Chinese population are lacking, and the distribution patterns of SSTR in normal pituitary and somatotrophic adenomas have not been reported. In this study, we examined the value of SSTR expression in predicting the short-term efficacy of SSA and the distribution patterns of SSTR in the normal pituitary and somatotrophic adenomas.

\section{Materials and Methods}

2.1. Patients and Samples. Normal pituitary samples with no clinical or pathological evidence of endocrine disorders ( $n=20$, normal pituitary group, NP group) were obtained via autopsy. Pathological examination excluded the presence of pituitary adenomas. We recruited 97 newly diagnosed and untreated patients with acromegaly at a tertiary referral center in East China from September 2008 to August 2013. Four patients were excluded from our study because of sparse adenoma tissue in the remaining specimens. Acromegaly was diagnosed according to clinical features including failure of $\mathrm{GH}$ suppression to below $1 \mu \mathrm{g} / \mathrm{L}$ in response to a $75 \mathrm{~g}$ oral glucose load, serum insulin-like growth factor 1 (IGF-1) levels above the age-matched reference range, and radiological evidence of pituitary tumors. Enhanced MRI was performed to identify the size and position of the pituitary adenomas. 93 patients were included in the statistical analysis with baseline clinical characteristics presented in Table 1. For these patients, the decision regarding primary treatment was made based on guidelines for acromegaly, clinical evaluation by a multidisciplinary pituitary tumor conference (consisting of endocrinologists, neurosurgeons, radiologists, and radiation therapists), and the patients' preference.

Of the 93 patients, 63 were pretreated with long-acting SSA (octreotide LAR or lanreotide SR, SA group) for 3 months prior to their surgeries, and the other 30 underwent surgery without medical pretreatment (direct surgery, DS group). Patients were evaluated after 3 months of SSA treatment. Random GH, IGF-1, and pituitary MRI were performed to evaluate the response to SSA. Biochemical response was defined as a posttreatment random $\mathrm{GH}$ on $\mathrm{GH}$ day curve $<2.5 \mu \mathrm{g} / \mathrm{L}$ or $>75 \%$ fall compared with the pretreatment random $\mathrm{GH}$ [16]. Oncological response was defined as tumor volume shrinkage $>20 \%$ [17].
TABLE 2: Clinical characteristics of the SA group before and after SSA treatment.

\begin{tabular}{lc}
\hline & SA group $(n=63)$ \\
\hline Baseline GH, $\mu \mathrm{g} / \mathrm{L}$ & $40.9(15.7-56.1)$ \\
Post-SSA GH, $\mu \mathrm{g} / \mathrm{L}$ & $7.9(2.9-34.6)$ \\
\% GH reduction & $69.6(32.4-90.5)$ \\
Baseline IGF-1 index & $3.0(2.6-3.6)$ \\
Post-SSA IGF-1 index & $2.0(1.3-2.5)$ \\
\% IGF-1 index reduction & $34.0(12.6-54.3)$ \\
\% tumor reduction & $23(10-45)$ \\
\hline
\end{tabular}

$\mathrm{SA}$, pretreatment with somatostatin analogues before surgery; $\mathrm{GH}$, growth hormone; IGF-1, insulin-like growth factor 1; SSA, somatostatin analogues. Data are median with interquartile range.

The Institutional Review Board at Huashan Hospital approved this study, and written informed consent was obtained from all patients before study entry.

2.2. Biochemical Measurements. IGF-1 was measured with Immulite 2000 solid-phase, which is an enzyme-labeled chemiluminescent immunometric assay (Siemens Healthcare Diagnostic Products Limited, UK). The normal ranges were age-dependent (1-6 years: $49-327 \mu \mathrm{g} / \mathrm{L}$; $7-11$ years: $57-551 \mu \mathrm{g} / \mathrm{L} ; 12-13$ years: $143-850 \mu \mathrm{g} / \mathrm{L} ; 14-16$ years: 220-996 $\mu \mathrm{g} / \mathrm{L} ; 17-18$ years: $163-731 \mu \mathrm{g} / \mathrm{L} ; 19-20$ years: $127-$ $483 \mu \mathrm{g} / \mathrm{L} ; 21-35$ years: $115-358 \mu \mathrm{g} / \mathrm{L} ; 36-50$ years: $94-$ $284 \mu \mathrm{g} / \mathrm{L}$; and > 50 years: $55-238 \mu \mathrm{g} / \mathrm{L}$ ) [18]. The IGF-1 index, a parameter that describes the level of the IGF-1 based on age (defined as the ratio of IGF-1 value to the maximum of reference ranges), was calculated for all patients. $\mathrm{GH}$ was measured by a two-site chemiluminescent immunometric assay using AutoDELFIA ${ }^{\circledR}$ hGH (PerkinElmer Life and Analytical Sciences, Wallac Oy).

2.3. Immunohistochemistry. The pituitary glands were fixed in $4 \%$ paraformaldehyde overnight and then embedded in paraffin. The sections (5 microns) were deparaffinized in methanol for 15 minutes and then treated with $10 \mathrm{mM}$ sodium citrate for 1 hour to unmask the antigen epitopes. Following this, endogenous peroxidase activity was blocked by treatment with $3 \%$ hydrogen peroxide for 30 minutes. Rabbit monoclonal antibodies for SSTR2 (1:100, ab134152, Abcam, US) and SSTR5 (1:100, ab109495, Abcam, US) were then examined as previously described [15]. The adenomas 


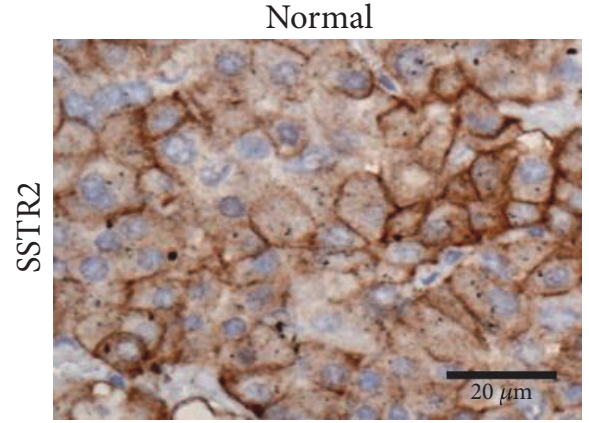

(a)

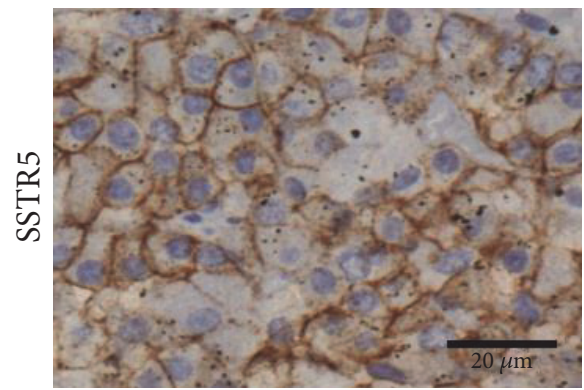

(d)

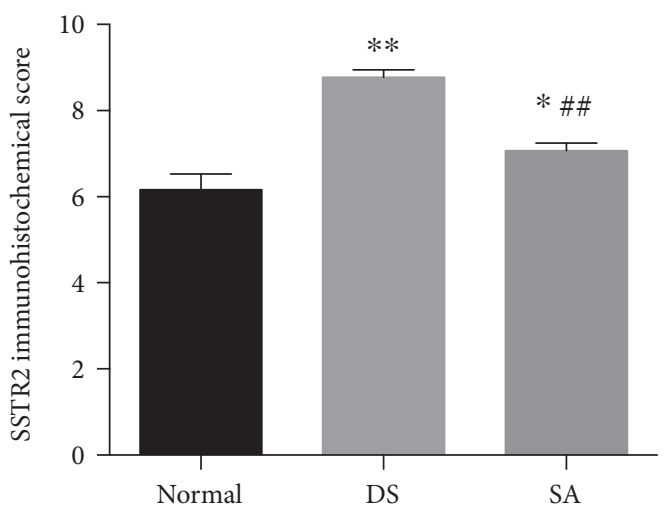

(g)
DS

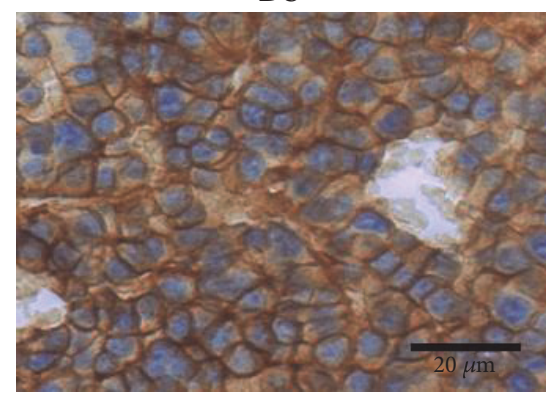

(b)

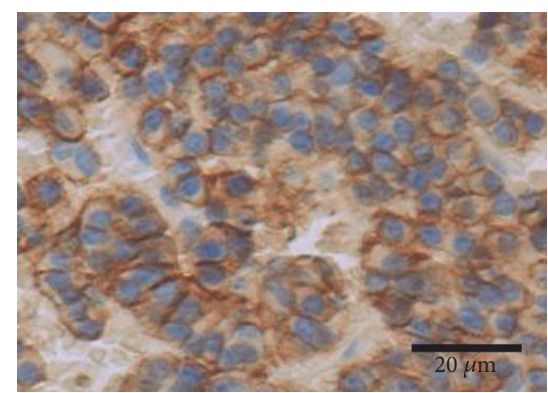

(e)
SA

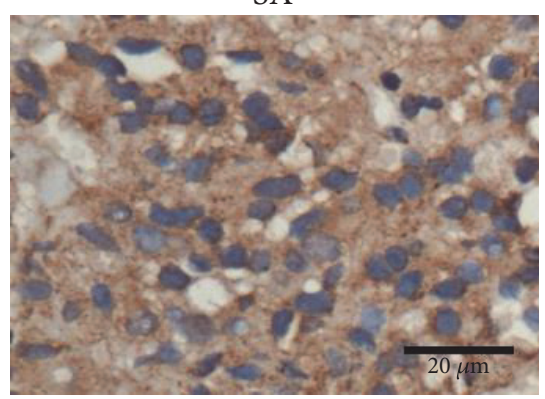

(c)

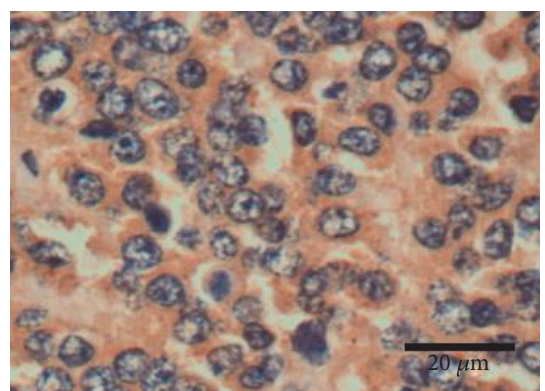

(f)

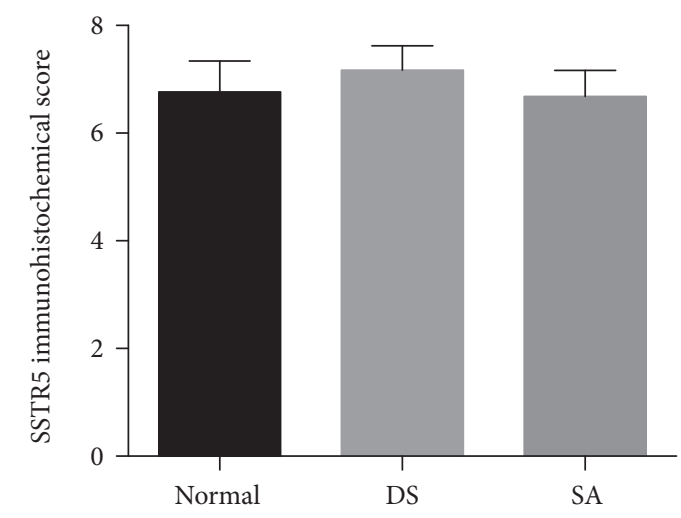

(h)

FIGURE 1: The protein expression patterns of SSTR2 and SSTR5: $(a-c)$ protein expression pattern of SSTR2 in the normal pituitary group, direct surgery group, and SSA pretreatment group; (d-f) SSTR5 expression pattern; and ( $g$ and $h)$ immunoreactive score of SSTR2 and SSTR5 for these three groups. Data are shown by mean \pm SEM. ${ }^{*} P<0.05$ and ${ }^{* *} P<0.01$ versus normal group; ${ }^{\# \#} P<0.01$ versus DS group.

were scored using the immunoreactive score (IRS) performed by two researchers who were blinded regarding the clinical data. The IRS $(0-12)$ is the product of the proportion of immunoreactive cells $(0,0 \% ; 1,<10 \% ; 2,10 \%-50 \% ; 3$, $51 \%-80 \%$; or $4,>80 \%)$ and the staining intensity ( 0 , no staining; 1 , weak; 2, moderate; and 3, strong). 10 pictures of each slice were analyzed, and the mean IRS was obtained for the following analysis.

2.4. Statistical Analysis. Data were presented as mean \pm (or median with interquartile range) for continuous variables that were normally (or not normally) distributed and as frequencies for categorical variables. The normal distribution of continuous parameters was analyzed by the Shapiro-Wilk test. After log transformation, the baseline
GH concentration, IGF-1 index, and tumor volume were normally distributed. Student's $t$-test was used to analyze 2 groups of normally distributed continuous variables before or after $\log$ transformation. The significance of the differences in mean values among different groups was evaluated using one-way ANOVA followed by the Tukey test. To analyze the correlations between the IRS and clinical parameters, the Pearson correlation coefficient or the Spearman rank correlation coefficient was calculated when variables were normally or not normally distributed, respectively. Following this, parametric or nonparametric significance testing was performed. Statistical analyses were performed using the statistical package SPSS for Mac Ver. 20.0 (SPSS Inc., Chicago, IL, USA). $P$ values less than 0.05 were considered to be statistically significant. 


\section{Results}

3.1. Basic Characteristics of the Cohort and the Differences between the SA and DS Groups. Table 1 shows the clinical information of the 93 patients, of which 59 are female and 34 are male. 63 patients received 3-month presurgical SSA treatment (SA group), and the other 30 patients underwent surgery directly without any pretreatment (DS group). The average age at diagnosis was $42 \mathrm{y}$ (IQR 35-53y). The mean baseline growth hormone level was $34.2 \mu \mathrm{g} / \mathrm{L}$ (IQR 13.5-57.9 $\mu \mathrm{g} / \mathrm{L}$ ), while the mean IGF-1 index was 2.9 (IQR 2.4-3.6). Data for tumor volumes were available in 77 cases, and the mean baseline tumor volume was $1900 \mathrm{~mm}^{3}$ (IQR 1000-2515 $\mathrm{mm}^{3}$ ).

Regarding the baseline characteristics of these two groups, data in Table 1 also showed that the IGF-1 index was higher in the SA group (3.0, IQR 2.6-3.6) than in the DS group (2.4, IQR 1.9-2.8) $(P=0.0054)$. However, baseline $\mathrm{GH}$ levels and adenoma volume were not different between these two groups (baseline GH levels: SA group, $40.9 \mu \mathrm{g} / \mathrm{L}$ (IQR, 15.7-56.1 $\mathrm{g} / \mathrm{L}$ ) versus DS group, $26.4 \mu \mathrm{g} / \mathrm{L}$ (IQR, $12.1-66.5 \mu \mathrm{g} / \mathrm{L}), P=0.22$; adenoma size: $\mathrm{SA}$ group, $2110 \mathrm{~mm}^{3}$ (IQR, $1000-2500 \mathrm{~mm}^{3}$ ) versus DS group, $1470 \mathrm{~mm}^{3}$ (IQR 249-6160 $\left.\mathrm{mm}^{3}\right), P=0.65$ ).

As shown in Table 2, after 3 months of long-acting SSA therapy, the mean posttreatment $\mathrm{GH}$ level was $7.9 \mu \mathrm{g} / \mathrm{L}$ (IQR, 2.9-34.6 $\mu \mathrm{g} / \mathrm{L}$ ), and the mean percentage fall of $\mathrm{GH}$ was $69.6 \%$ (IQR, 32.4-90.5\%). The mean posttreatment IGF-1 index was 2.0 (IQR, 1.3 2.5), while the mean percentage reduction of the IGF-1 index was $34.0 \%$ (IQR, $12.6-54.3 \%)$. The mean percentage reduction in tumor volume was $23.0 \%$ (IQR, 10.0-45.0\%).

3.2. The Protein Expression Pattern of SSTR2 and SSTR5. In order to detect the expression pattern of SSTR2 and SSTR5 in the normal pituitary and adenomas from both the DS group and SA group, we performed IHC analysis by using rabbit monoclonal antibodies against these two receptors. SSTR2 and SSTR5 from normal patients and the DS group exhibited predominantly membranous expression (Figures 1(a)-1(e)), while in the SA group, the expression of these two receptors was mainly localized in the cytoplasm (Figures 1(c) and 1(f)). Compared to the normal pituitary, the IRS of SSTR2 in the DS group was much higher (6 versus 9, IQR, 3.5-8.4 versus 7.2-10.3, resp., $P<0.01)$. However, pretreated adenomas (SA group) had a significantly lower score (8, IQR, 4.6-9.7) than those in the DS group $(9$, IQR, 7.2-10.3) $(P<0.01)$ (Figures 1(a)-1(c), 1(g)). Interestingly, there was no difference in the SSTR5 expression between the normal group (6, IQR, 5.0-8.6), DS group (6, IQR, 4.8-9.3), and SA group (6, IQR, 3.4-9.3) $(F=0.36, \quad P=0.69)$ (Figures 1(d)-1(f) and 1(h)).

3.3. Correlation between the Baseline Biomedical Characteristics of SA, DS, and the Entire Cohort and the Expression of SSTR2 and SSTR5. As Table 3 shows, in the SA group, there was a negative correlation between the SSTR2 score and the pretreatment GH levels $(r=-0.312$,
TABLE 3: Correlation between the baseline biomedical characteristics of SA, DS, and the entire cohort and the expression of SSTR2 and SSTR5.

\begin{tabular}{lccc}
\hline & Baseline GH & $\begin{array}{c}\text { Baseline } \\
\text { IGF-1 index }\end{array}$ & $\begin{array}{c}\text { Baseline tumor } \\
\text { volume }\end{array}$ \\
\hline SA group & & & \\
SSTR2 & $r=-0.312$ & $r=0.287$ & $r=-0.079$ \\
& $P=0.015$ & $P=0.043$ & $P=0.561$ \\
SSTR5 & $r=0.068$ & $r=-0.001$ & $r=-0.032$ \\
& $P=0.645$ & $P=0.997$ & $P=0.831$ \\
\hline DS group & & & \\
SSTR2 & $r=-0.5$ & $r=0.099$ & $r=-0.238$ \\
& $P=0.791$ & $P=0.61$ & $P=0.342$ \\
SSTR5 & $r=-0.159$ & $r=0.1$ & $r=-0.195$ \\
& $P=0.402$ & $P=0.644$ & $P=0.342$ \\
\hline Entire cohort & & & $r=-0.125$ \\
SSTR2 & $P=-0.228$ & $r=0.019$ & $P=0.284$ \\
& $P=0.032$ & $P=0.866$ & $r=-0.108$ \\
SSTR5 & $r=-0.029$ & $r=-0.229$ & $P=0.391$ \\
\hline
\end{tabular}

SA, pretreatment with somatostatin analogues before surgery; DS, direct surgery group; GH, growth hormone; IGF-1, insulin-like growth factor 1; SSTR, somatostatin receptor. The Pearson correlation coefficient was used to analyze the correlations between the immunoreactive score (IRS) and clinical parameters.

$P=0.015)$, which suggested that a low SSTR2 score was associated with increased GH secretion. Moreover, this negative correlation was also found in the entire cohort $(r=-0.228$, $P=0.032$ ), but not in the DS group.

The baseline IGF-1 index level was correlated with the SSTR2 score $(r=0.287, P=0.043)$ in the SA group. There were no significant correlations between the SSTR2 score and the baseline tumor volume in any group. As for SSTR5, there were no significant correlations between it and the baseline biochemical indexes in these groups.

3.4. Correlation between the Clinical Biomedical Characteristics in SA Group and the Expression of SSTR2 and SSTR5. As shown in Table 4, there was a negative correlation between the SSTR2 score and post-SSA GH levels $(r=-0.353$, $P=0.006)$, although no significant correlation was found between the SSTR2 score and the GH reduction or the $\mathrm{GH}$ reduction percentage (Table 4 and Figure 2(a)). The relative IGF-1 index reduction (IGF-1 index reduction ratio) and the SSTR2 score were significantly positively correlated ( $r=0.403, P=0.004$, Table $4 ; r=0.413, P=0.003$, Figure 2(b), resp.), and there was a trend suggesting that the SSTR2 score was negatively correlated with the posttreatment IGF-1 index $(r=-0.227, P=0.094)$. Notably, the reduction of tumor volume was significantly correlated with the SSTR2 score $(r=0.367, P=0.005$, Figure 2(c)). These results suggested that the SSTR2 IHC score could predict the short-term biomedical response and tumor shrinkage with SSA treatment. 
TABLE 4: Correlation between the clinical biomedical characteristics in SA group and the expression of SSTR2 and SSTR5.

\begin{tabular}{|c|c|c|c|c|c|c|c|}
\hline $\begin{array}{l}\text { SA } \\
\text { group }\end{array}$ & $\begin{array}{c}\text { Post-SSA } \\
\text { GH } \\
\end{array}$ & $\begin{array}{c}\mathrm{GH} \\
\text { reduction }\end{array}$ & $\begin{array}{l}\% \mathrm{GH} \\
\text { reduction }\end{array}$ & $\begin{array}{c}\text { Post-SSA IGF-1 } \\
\text { index }\end{array}$ & $\begin{array}{l}\text { IGF-1 index } \\
\text { reduction }\end{array}$ & $\begin{array}{c}\% \text { IGF-1 index } \\
\text { reduction }\end{array}$ & $\begin{array}{l}\% \text { tumor } \\
\text { reduction }\end{array}$ \\
\hline \multirow{2}{*}{ SSTR2 } & $r=-0.353$ & $r=-0.14$ & $r=0.163$ & $r=-0.227$ & $r=0.403$ & $r=0.413$ & $r=0.367$ \\
\hline & $P=0.006$ & $P=0.342$ & $P=0.267$ & $P=0.094$ & $P=0.004$ & $P=0.003$ & $P=0.005$ \\
\hline \multirow{2}{*}{ SSTR5 } & $r=-0.044$ & $r=0.006$ & $r=0.068$ & $r=-0.244$ & $r=0.222$ & $r=0.292$ & $r=0.17$ \\
\hline & $P=0.765$ & $P=0.97$ & $P=0.678$ & $P=0.099$ & $P=0.153$ & $P=0.057$ & $P=0.263$ \\
\hline
\end{tabular}

SA, pretreatment with somatostatin analogues before surgery; GH, growth hormone; IGF-1, insulin-like growth factor 1; SSA, somatostatin analogues; SSTR, somatostatin receptor. The Pearson correlation coefficient was used to analyze the correlations between the immunoreactive score (IRS) and clinical parameters.

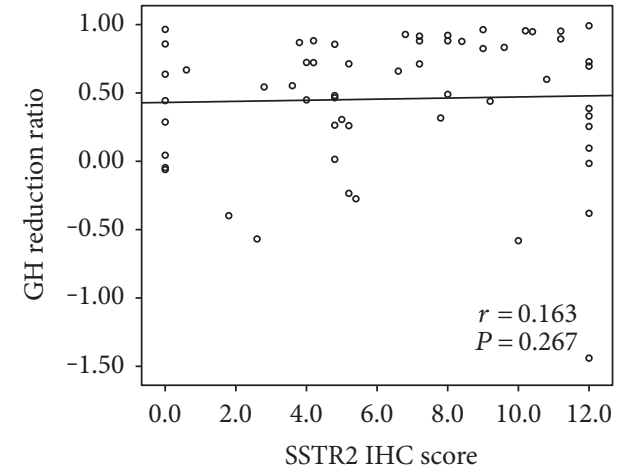

(a)

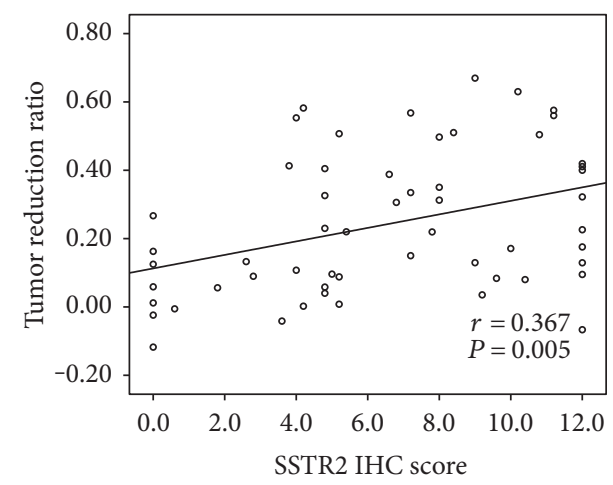

(c)

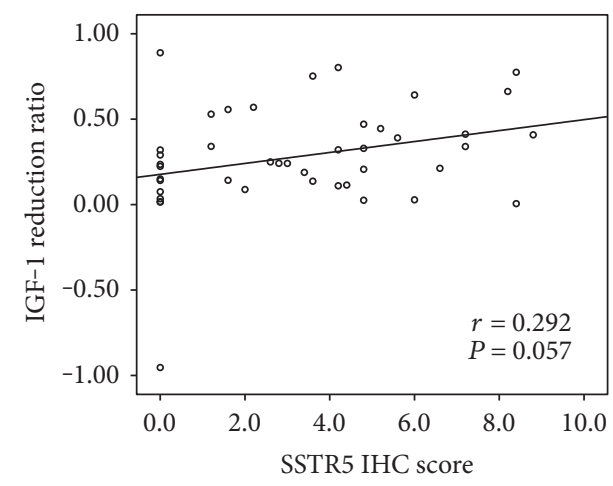

(e)

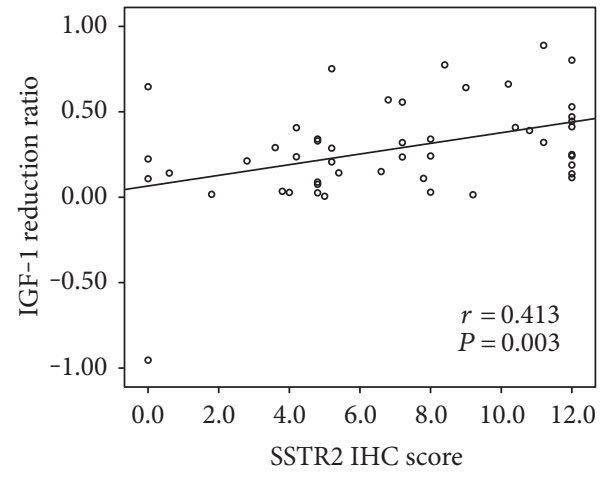

(b)

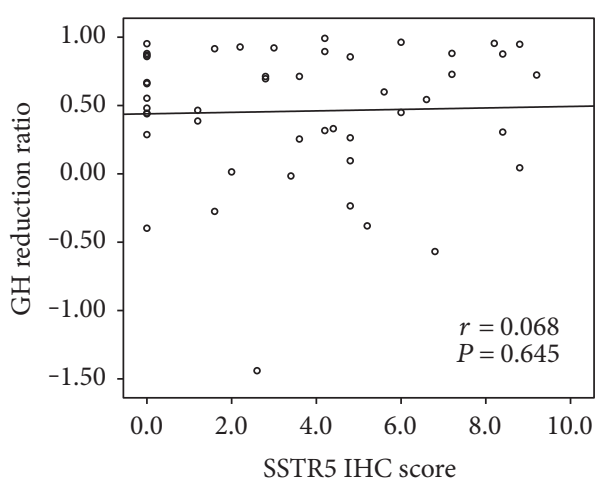

(d)

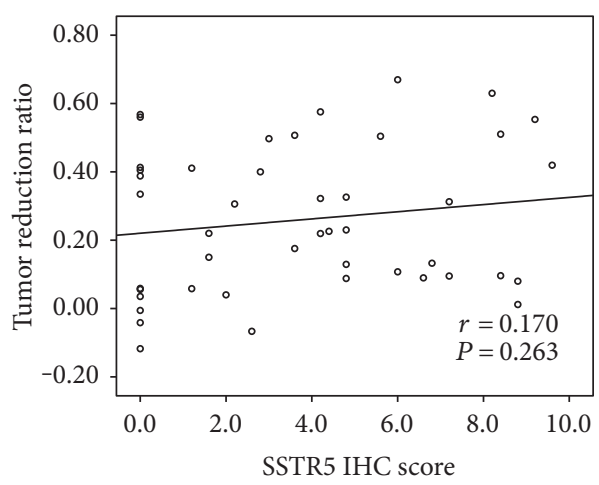

(f)

FIGURE 2: Correlation between GH (IGF-1 or tumor volume) reduction ratio and the expression of SSTR2 (or SSTR5): (a-c) correlation between the SSTR2 IRS score with GH reduction ratio, IGF-1 reduction ratio or tumor volume reduction ratio, respectively, and (d-f) correlation between SSTR5 IRS score with the same three indexes. 
There were no significant correlations between the SSTR5 score and the clinical indexes, including baseline post-SSA GH, GH reduction, $\mathrm{GH}$ reduction ratio, IGF-1 index reduction, and tumor reduction (Table 4 and Figures 2(d)-2(f)). However, there was a trend suggesting that SSTR5 score was negatively correlated with posttreatment IGF-1 index $(r=-0.244, P=0.099)$, and the IHC score of SSTR5 tended to be positively correlated with the IGF-1 reduction percentage $(r=0.292, P=0.057)$.

\section{Discussion}

In this study, we demonstrated for the first time in Chinese patients that the expression pattern of SSTR2 was different in the normal pituitary compared to somatotrophic adenomas. Moreover, SSTR2 expression was significantly lower in adenomas that were exposed to octreotide prior to surgery than those that did not receive pretreatment. We also determined that SSTR2 expression was correlated with the shortterm biochemical and tumor volume response to SSA.

Somatostatin analogues (SSA) have been identified as the first line of medications in treating acromegalic patients $[4,19]$. However, the response to SSA treatment is highly variable: some patients achieve full biochemical remission and a considerable reduction of tumor volume, whereas others are resistant (see Table 1 in Supplementary Material available online at https://doi.org/10.1155/2017/ 9606985) [13]. It is therefore urgent to find a way of identifying patients who may benefit from the SSA treatment.

Since SSTR2 and SSTR5 are the predominantly bound receptors for SSA, plenty of studies have focused on the predictive values of SSTR2 and SSTR5 expression. Results regarding the correlation between SSTR2 and SSTR 5 mRNA and/or protein level and in vivo response to SSA have been subject to debate so far $[7,9,14,20]$. Although there is a positive correlation between SSTR2 mRNA expression and the response of SSA, the mRNA level does not necessarily reflect the protein level or the functional activity due to translational and posttranslational regulation of protein synthesis [21]. Moreover, before the development of monoclonal antibodies against SSTR2 and SSTR5, IHC studies have mainly been limited to analyze the expression of only SSTR2 [22], partly because it is the most relevant receptor for SSA binding and the polyclonal antibodies against other SSTR subtypes are unsatisfactory [15].

Our results showed that the SSTR2 protein expression, but not the SSTR5, was much higher in acromegalic patients than normal subjects. This was supported by a study from Neto et al. [23] who reported that the mRNA expression of SSTR2 in somatotropinomas was higher than in the normal pituitary. Moreover, we also observed a significantly lower SSTR2 score after SSA treatment, whereas expression of SSTR5 remained unchanged. This is consistent with the previous reports from Casar-Borota, Fougner, and Plockinger [15, 22, 24].

The reason why SSA treatment could reduce SSTR2 IHC scores may be the receptor internalization of SSTR2. Basic research has demonstrated that SSTR2 could translocate from the cell membrane to the cytoplasm after SSA treatment
[25-27]. Our IHC results also verified this phenomenon. In the normal pituitary and DS groups, the SSTR2 exhibited a predominantly membranous expression pattern. However, in the SA group, this receptor was more frequently localized in the cytoplasm. Taken together, these data suggested that SSA altered the expression and distribution pattern of SSTR2.

In summary, the IHC score of SSTR2 is a valuable indicator for the prediction of both biochemical and tumor size response to SSA and could be a clinically useful index to predict the short-term efficacy of SSA treatment in acromegalic patients. However, due to the small sample size of patients involved, further studies should be planned in order to investigate and validate our results. In this study, we performed the IHC test using monoclonal anti-SSTR2 and SSTR5 antibodies. Consistent with a previous study [15], SSTR2 expression, but not SSTR5, was a valuable indicator in predicting the short-term efficacy of SSA in acromegalic patients.

\section{Conflicts of Interest}

The authors of this manuscript have no potential conflict of interests to declare.

\section{Authors' Contributions}

Wenjuan Liu, Lina Xie, and Min He contributed equally to this work.

\section{Acknowledgments}

This study was supported by the grants from the Natural Science Foundation of China (81370938, 81471041), Shanghai Municipal Commission of Health and Family Planning (XYQ2011002), and Shanghai Science and Technology Commission (11PJ1402000).

\section{References}

[1] G. Guinto, M. Abdo, E. Zepeda, N. Arechiga, and M. Mercado, "Acromegaly: role of surgery in the therapeutic armamentarium," International Journal of Endocrinology, vol. 2012, Article ID 306094, p. 13, 2012.

[2] S. M. Carlsen, J. Svartberg, T. Schreiner et al., "Six-month preoperative octreotide treatment in unselected, de novo patients with acromegaly: effect on biochemistry, tumour volume, and postoperative cure," Clinical Endocrinology, vol. 74, no. 6, pp. 736-743, 2011.

[3] M. Mercado, F. Borges, H. Bouterfa et al., "A prospective, multicentre study to investigate the efficacy, safety and tolerability of octreotide LAR (long-acting repeatable octreotide) in the primary therapy of patients with acromegaly," Clinical Endocrinology, vol. 66, no. 6, pp. 859-868, 2007.

[4] A. Ben-Shlomo and S. Melmed, "Somatostatin agonists for treatment of acromegaly," Molecular and Cellular Endocrinology, vol. 286, no. 1-2, pp. 192-198, 2008.

[5] A. Colao, R. S. Auriemma, and R. Pivonello, "The effects of somatostatin analogue therapy on pituitary tumor volume in patients with acromegaly," Pituitary, vol. 19, no. 2, pp. 210-221, 2016. 
[6] P. Legovini, E. De Menis, D. Billeci, B. Conti, P. Zoli, and N. Conte, "111Indium-pentetreotide pituitary scintigraphy and hormonal responses to octreotide in acromegalic patients," Journal of Endocrinological Investigation, vol. 20, no. 7, pp. 424-428, 1997.

[7] M. R. Gadelha, L. Kasuki, and M. Korbonits, "Novel pathway for somatostatin analogs in patients with acromegaly," Trends in Endocrinology and Metabolism, vol. 24, no. 5, pp. 238-246, 2013.

[8] M. Sheppard, M. D. Bronstein, P. Freda et al., "Pasireotide LAR maintains inhibition of GH and IGF-1 in patients with acromegaly for up to 25 months: results from the blinded extension phase of a randomized, double-blind, multicenter, phase III study," Pituitary, vol. 18, no. 3, pp. 385-394, 2015.

[9] L. E. Wildemberg, L. V. Neto, D. F. Costa et al., "Low somatostatin receptor subtype 2, but not dopamine receptor subtype 2 expression predicts the lack of biochemical response of somatotropinomas to treatment with somatostatin analogs," Journal of Endocrinological Investigation, vol. 36, no. 1, pp. 38-43, 2013.

[10] M. Wang, M. Shen, W. He et al., "The value of an acute octreotide suppression test in predicting short-term efficacy of somatostatin analogues in acromegaly," Endocrine Journal, vol. 63, no. 9, pp. 819-834, 2016.

[11] E. Verrua, E. Ferrante, M. Filopanti et al., "Reevaluation of acromegalic patients in long-term remission according to newly proposed consensus criteria for control of disease," International Journal of Endocrinology, vol. 2014, Article ID 581594, p. 8, 2014.

[12] T. R. Bandgar, V. Sarathi, V. Shivane, N. Bansode, P. S. Menon, and N. S. Shah, "The value of an acute octreotide suppression test in predicting response to long-term somatostatin analogue therapy in patients with acromegaly," Journal of Postgraduate Medicine, vol. 56, no. 1, pp. 7-11, 2010.

[13] P. U. Freda, L. Katznelson, A. J. van der Lely, C. M. Reyes, S. Zhao, and D. Rabinowitz, "Long-acting somatostatin analog therapy of acromegaly: a meta-analysis," The Journal of Clinical Endocrinology and Metabolism, vol. 90, no. 8, pp. 4465-4473, 2005.

[14] G. F. Taboada, R. M. Luque, L. V. Neto et al., "Quantitative analysis of somatostatin receptor subtypes (1-5) gene expression levels in somatotropinomas and correlation to in vivo hormonal and tumor volume responses to treatment with octreotide LAR," European Journal of Endocrinology, vol. 158, no. 3, pp. 295-303, 2008.

[15] O. Casar-Borota, A. Heck, S. Schulz et al., "Expression of SSTR2a, but not of SSTRs 1, 3, or 5 in somatotroph adenomas assessed by monoclonal antibodies was reduced by octreotide and correlated with the acute and long-term effects of octreotide," The Journal of Clinical Endocrinology and Metabolism, vol. 98, no. 11, pp. E1730-E1739, 2013.

[16] A. Heck, K. E. Emblem, O. Casar-Borota, J. Bollerslev, and G. Ringstad, "Quantitative analyses of T2-weighted MRI as a potential marker for response to somatostatin analogs in newly diagnosed acromegaly," Endocrine, vol. 52, no. 2, pp. 333-343, 2016.

[17] P. J. Caron, J. S. Bevan, S. Petersenn et al., "Tumor shrinkage with lanreotide Autogel $120 \mathrm{mg}$ as primary therapy in acromegaly: results of a prospective multicenter clinical trial," The Journal of Clinical Endocrinology and Metabolism, vol. 99, no. 4, pp. 1282-1290, 2014.
[18] G. Lugo, L. Pena, and F. Cordido, "Clinical manifestations and diagnosis of acromegaly," International Journal of Endocrinology, vol. 2012, Article ID 540398, p. 10, 2012.

[19] S. Melmed, "Acromegaly pathogenesis and treatment," The Journal of Clinical Investigation, vol. 119, no. 11, pp. 3189-3202, 2009.

[20] D. Ferone, W. W. de Herder, R. Pivonello et al., "Correlation of in vitro and in vivo somatotropic adenoma responsiveness to somatostatin analogs and dopamine agonists with immunohistochemical evaluation of somatostatin and dopamine receptors and electron microscopy," The Journal of Clinical Endocrinology and Metabolism, vol. 93, no. 4, pp. 1412-1417, 2008.

[21] L. E. Wildemberg, L. Vieira Neto, D. F. Costa et al., "Validation of immunohistochemistry for somatostatin receptor subtype 2A in human somatotropinomas: comparison between quantitative real time RT-PCR and immunohistochemistry," Journal of Endocrinological Investigation, vol. 35, no. 6, pp. 580-584, 2012.

[22] S. L. Fougner, O. C. Borota, J. P. Berg, J. K. Hald, J. RammPettersen, and J. Bollerslev, "The clinical response to somatostatin analogues in acromegaly correlates to the somatostatin receptor subtype 2a protein expression of the adenoma," Clinical Endocrinology, vol. 68, no. 3, pp. 458-465, 2008.

[23] L. V. Neto, O. Machado Ede, R. M. Luque et al., "Expression analysis of dopamine receptor subtypes in normal human pituitaries, nonfunctioning pituitary adenomas and somatotropinomas, and the association between dopamine and somatostatin receptors with clinical response to octreotideLAR in acromegaly," The Journal of Clinical Endocrinology and Metabolism, vol. 94, no. 6, pp. 1931-1937, 2009.

[24] U. Plockinger, S. Albrecht, C. Mawrin et al., "Selective loss of somatostatin receptor 2 in octreotide-resistant growth hormone-secreting adenomas," The Journal of Clinical Endocrinology and Metabolism, vol. 93, no. 4, pp. 1203-1210, 2008.

[25] S. Lesche, D. Lehmann, F. Nagel, H. A. Schmid, and S. Schulz, "Differential effects of octreotide and pasireotide on somatostatin receptor internalization and trafficking in vitro," The Journal of Clinical Endocrinology and Metabolism, vol. 94, no. 2, pp. 654-661, 2009.

[26] R. Cescato, S. Schulz, B. Waser et al., "Internalization of sst2, sst3, and sst5 receptors: effects of somatostatin agonists and antagonists," The Journal of Nuclear Medicine, vol. 47, no. 3, pp. 502-511, 2006.

[27] A. Colao, R. S. Auriemma, G. Lombardi, and R. Pivonello, "Resistance to somatostatin analogs in acromegaly," Endocrine Reviews, vol. 32, no. 2, pp. 247-271, 2011. 


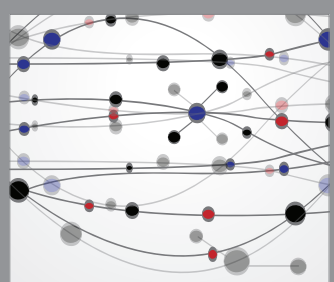

The Scientific World Journal
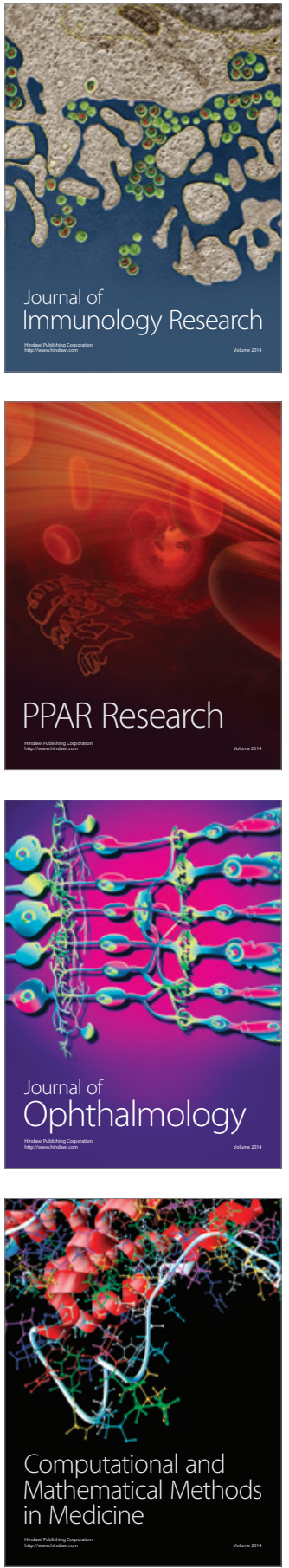

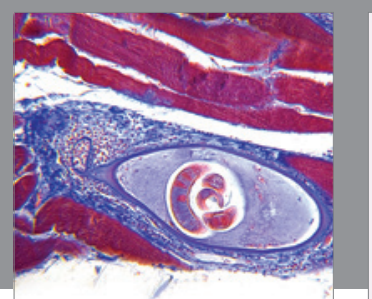

Gastroenterology Research and Practice
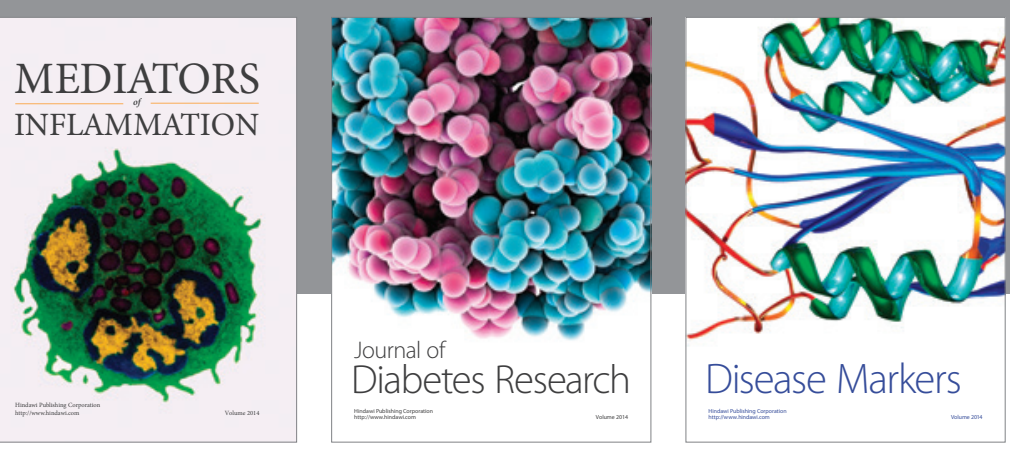

Disease Markers

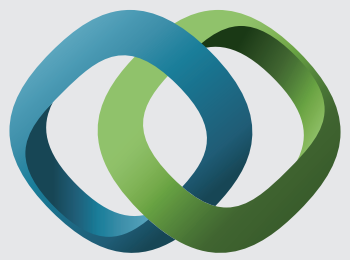

\section{Hindawi}

Submit your manuscripts at

https://www.hindawi.com
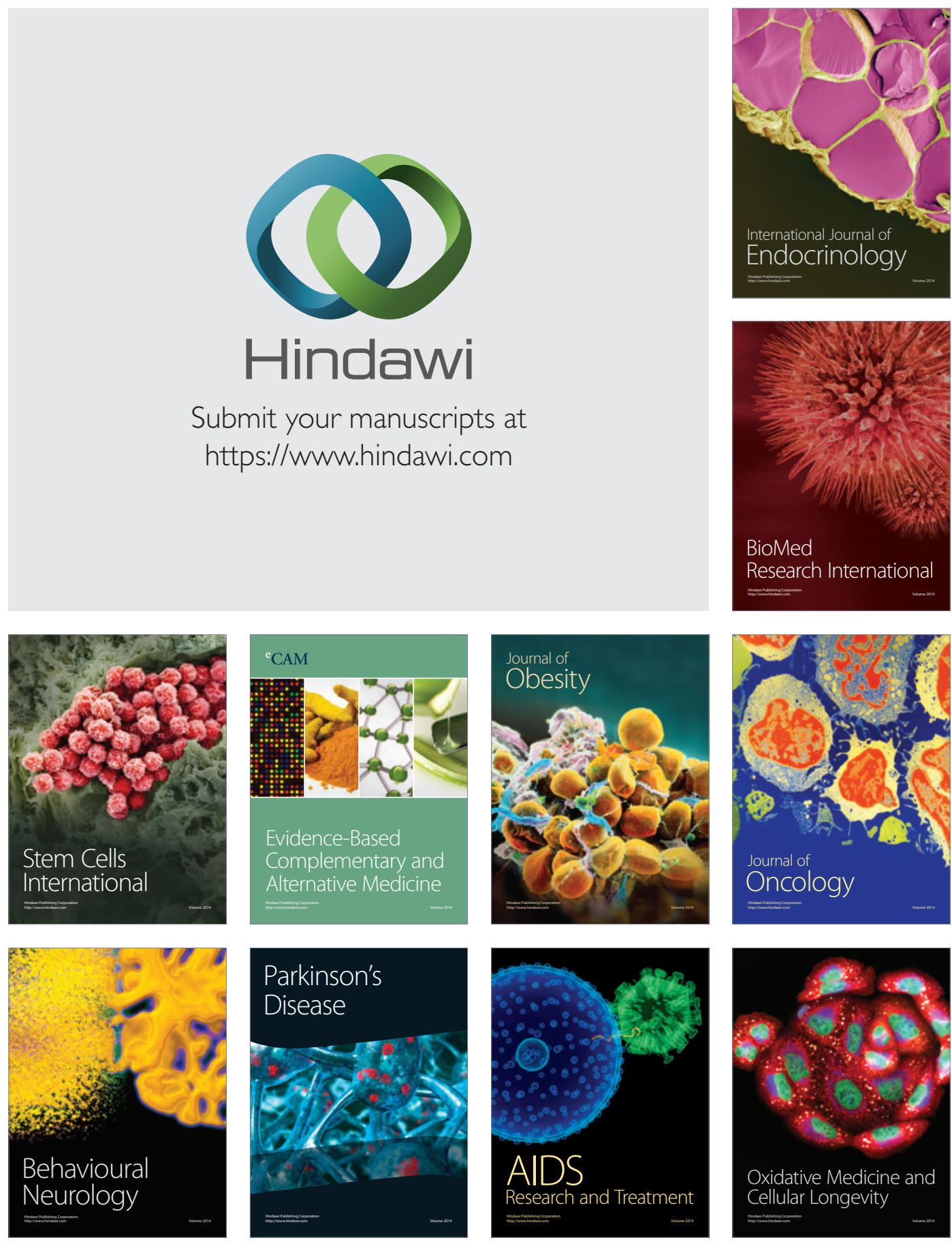